\title{
11. Lässt sich die Pyroelectricität der Krystalle vollständig auf piëzoelectrische Wirkungen zurückführen? Von W. Voigt.
}

(Mitgetheilt aus den Göttinger Nachrichten, Heft 2. 1898.)

\section{Einleitung.}

Es ist eine bereits von den Entdeckern der Erscheinungen der Piëzoelectricität, den Herren J. und P. Curie ${ }^{1}$ ), ausgesprochene Vermuthung, dass die sogenannte Pyroelectricität in Wahrheit auf piëzoelectrischen Erregungen beruhen möchte, d.h., dass die Temperaturänderung eines Krystalles nur deshalb electricitäterregend wirkt, weil sie ihn deformirt. Diese Vermuthung hat später besonders Hr. Röntgen ${ }^{2}$ ) auf Grund seiner Beobachtungen mit grossem Nachdruck vertreten.

Ihre exacte Prüfung erfordert ausser quantitativen Bestimmungen der bezüglichen Erscheinungen eine vollständige Entwickelung der Theorie der Piëzoelectricität, da nur mit Hülfe einer solchen die erregende Wirkung einer thermischen Deformation zu derjenigen einer mechanischen Deformation in Beziehung gebracht werden kann. Diese allgemeine Theorie habe ich vor nunmehr acht Jahren veröffentlicht. ${ }^{3}$ ) Für eine grosse Zahl von Krystallgruppen ergiebt sich indessen die Richtigkeit der oben dargestellten Vermuthung ohne die Hülfe von Beobachtungen schon aus den ersten Grundannahmen jener Theorie mittelst einer einfachen Symmetriebetrachtung. Nennt man nämlich wahre pyroelectrische krregung diejenige, welche in einem Krystalle durch eine gleichförmige Erwärmung bei aufgehobener Deformation hervorgerufen wird, dann ist von vornherein' klar, dass eine solche nur bei Krystallen auftreten kann, die eine einzelne krystallographisch ausgezeichnete Richtung besitzen, z. B, eine einzigartige polare Symmetrieaxe. 
Schreibt man also die Componenten $a, b, c$ des dielectrischen Momentes der Volumeneinheit in der Form

$$
\begin{gathered}
a=l\left(x_{x}, y_{y}, z_{z}, y_{z}, z_{x}, x_{y}, \tau\right), b=G\left(x_{x}, y_{y}, z_{z}, y_{z}, z_{x}, x_{y}, \tau\right), \\
c=H\left(x_{x}, y_{y}, z_{z}, y_{z}, z_{x}, x_{y}, \tau\right),
\end{gathered}
$$

wobei $F, G, H$ beliebige Functionen der Deformationsgrössen $x_{x}, \ldots x_{y}$ und der Temperaturänderung $\tau$ bezeichnen, so ist für jene Krystalle jedenfalls

$$
a=F(0,0,0,0,0,0, \tau)=0 \text { etc. }
$$

Die Deformationsgrössen $x_{x}, \ldots x_{y}$ sind nun in allen praktisch realisirbaren Fällen äusserst kleine Zahlen, sodass man bei der Entwickelung der Functionen $F, G, I I$ nach ihnen stets mit den ersten Gliedern abbrechen kann. Beschränkt man sich auch auf Temperaturänderungen $\tau$ von derselben Grössenordnung, so wird

$$
a=F_{1}^{\prime} x_{x}+F_{2} y_{y}+F_{3} z_{z}+F_{4}^{\prime} y_{z}+F_{5}^{\prime} z_{x}+F_{6} x_{y}+F_{7} \tau \text {, etc., }
$$

wo nunmehr die $F_{h}$, . Constanten bezeichnen.

Für die Krystalle ohne eine einzelne ausgezeichnete Richtung sind aber nach dem soeben Gesagten $F_{7}, G_{7}, I_{7}$ gleich Null, und dies zeigt, dass bei ihnen jede directe Einwirkung der I'emperatur auf die electrische Erregung ausgeschlossen ist. Wenn hier also durch eine Erwärmung ein electrisches Moment hervorgerufen wird, so ist dies stets eine piëzoelectrische Wirkung; die anscheinend vorhandene Pyroelectricität ist, wie wir sagen wollen, eine falsche. Es dürfte nützlich sein, dies zur Vermeidung von Missverständnissen nachdrücklich zu betonen.

Die genauere Untersuchung zeigt übrigens, dass solche Krystalle bei gleichförmiger Erwärmung auch dann keine electrische Erregung zeigen, wenn die jene begleitenden Deformationen ungehindert zu Stande kommen; sie reagiren vielmehr nur auf ungleichförmige Erwärmungen. Es härigt dies, wie die durchgeführte Theorie zeigt, aufs Engste damit zusammen, dass jene Krystalle nicht durch einen allseitig gleichen Druck, wohl aber durch einen mit der Richtung variirenden electrisch erregt werden. ${ }^{1}$ )

1) In dem allgemeineren Falle beliebig grosser Temperaturänderungen liegt die Sache, beiläufig gesagt, etwas anders. Hier würde zu schreiben sein

$$
a=F_{0}+F_{1} x_{x}+\ldots F_{8} x_{y} \text { ete. }
$$


Soweit die Frage nach der Identität von Pyro- und Piëzoelectricität, oder, - was damit nach dem Vorstehenden identisch ist, - diejenige nach der Existenz wahrer Pyroelectricität hierdurch nicht bereits erledigt ist, $d$. $h$. also bei Krystallen, die einzelne krystallographisch ausgezeichnete Richtungen besitzen, ist sie nicht ohne Heranziehung der Beobachtung $z u$ entscheiden. Theoretisch am einfachsten wäre diese Entscheidung so zu treffen, dass man einen Krystall erwärmte und zugleich durch ein System geeigneter äusserer Drucke seine Deformation aufhöbe; die ganze hierbei entstehende electrische Frregung würde dann wahre Pyroelectricität darstellen. Indessen bietet dieser Weg unüberwindliche praktische Schwierigkeiten, und man ist daher gezwungen, einen Umweg einzuschlagen, der eine Combination von Theorie und Beobachtung benutzt.

Gelegentlich unserer gemeinsamen Beobachtungen zur Prüfung der von mir gegebenen Formeln und zur Bestimmung der piëzoelectrischen Constanten für Quarz und Turmalin haben mein College E. Riecke und ich auch die eben angeregte Frage untersucht. ${ }^{1}$ ) Turmalin ist ja nicht nur ein Krystall mit einer ausgezeichneten Richtung ( - jede der beiden Seiten seiner polaren Hauptaxe ist einzigartig -), sondern auch einer der am stärksten auf eine gleichförmige Erwärmung reagirenden.

Die von uns angestellte Ueberlegung war diese. Durch Beobachtungen von Riecke war die Zunahme des specifischen electrischen Momentes bei einer Temperatursteigerung um $1^{\circ} \mathrm{C}$. gefunden; durch die erwähnten Beobachtungen von Riecke und mir, bei Benutzung der von mir erhaltenen Werthe der Elasticitätsmoduln und bei Anwendung der von mir gegebenen allgemeinen Formeln waren die piëzoelectrischen Constanten des Turmalines bekannt, und damit war die Berechnung der Aenderungen des specifischen electrischen Mo-

wobei jetzt die $F_{h}$ Functionen der vorhandenen Temperatur darstellen. Für die oben charakterisirten Krystalle wäre dann $F_{0}, G_{0}, H_{0}$ gleich Null; bei ihnen stellt sich also die Wirkung einer Erwärmung auf das electrische Moment als eine Veränderlichkeit der piëzoelectrischen Erregung mit der Temperatur dar.

1) E. Riecke u. W. Voigt, Wied. Ann. 45. p. 523. 1892. 
mentes bei beliebigen Deformationen ermöglicht. Bestimmte man noch die Deformation, welche der Turmalin bei einer Temperatursteigerung um $1^{\circ} \mathrm{C}$. erleidet, mit Hülfe der von anderen Autoren mitgetheilten Werthe seiner thermischen Dilatationsconstanten, so konnte man die electrische Erregung berechnen, die er bei diesem Vorgange zeigen würde, wenn wirklich nach der Curie'schen Hypothese nur die Deformation für die Erregung maassgebend wäre. Ihre Vergleichung mit der von Riecke erhaltenen Zahl musste dann eine Entscheidung über jene Hypothese liefern.

Unsere Rechnung ergab eine uns überraschende Uebereinstimmung zwischen den zu vergleichenden Werthen, - die Differenz zwischen ihnen betrug nur etwa zehn Procent und wir waren geneigt, wie wir damals auch aussprachen, derselben ein entscheidendes Gewicht beizulegen. Indessen liegt doch der Verdacht nahe, dass bei dem Resultat ein Zufall mitgewirkt habe. Die Anwendung der Theorie erfordert die Combination einer sehr erheblichen Anzahl von auf verschiedenen Wegen gewonnenen physikalischen Constanten, und, ganz abgesehen von der Unsicherheit einer jeden einzelnen Bestimmung, erregt es Bedenken, dass diese Constanten nicht sämnitlich an demselben Krystall gemessen waren, und dass gerade die, welche von anderen herübergenommen wurden, von einem Individuum zum andern recht stark zu variiren scheinen. Es sind dies die eine Constante der Pyroelectricität und die zwei der thermischen Dilatation, während die sechs Constanten der Elasticität und die vier der Piëzoelectricität sämmtlich für denselben Krystall bestimmt worden waren.

Die Bedenken gegen die entscheidende Kraft unserer früheren Rechnung wurden bei mir noch verstärkt durch die Folgerungen, die sich für die angeregte Frage aus der von mir vertretenen Anschauung über das Zustandekommen der Pyro- und Piëzoelectricität ${ }^{1}$ ) ergeben. Wenn diese Vorgänge, wie ich vermuthe, auf der Dislocation der electrischen Atome oder Pole beruhen, welche in den Molecülen der Krystalle in einer den Symmetrieverhältnissen entsprechenden Anordnung gruppirt sind (bez. um derartige Gleichgewichtslagen schwingen),

1) W. Voigt, Wied. Ann. 51. p. 659. 1894. 
und wenn solche Dislocationen durch Deformationen der Volumenelemente bewirkt werden können, so erscheint es nach den Vorstellungen der kinetischen Wärmetheorie als nahezu selbstverständlich, dass auch eine blosse Temperaturveränderung ohne begleitende Deformation die Anordnung der electrischen Pole - natürlich ohne deren Symmetrie zu tangiren - ändern wird. Auf Grund einer solchen Vorstellung wird man also die Existenz wahrer Pyroelectricität jedenfalls erwarten müssen, und in dem Falle, dass dieselbe nicht wahrnehmbar ist, nicht auf ein absolutes Fehlen, sondern nur auf eine sehr geringe Stärke der betreffenden Erregung schliessen.

Diesen Erwägungen entsprechend habe ich in einer späteren Arbeit ${ }^{1}$ ), welche die allgemeine Thermodynamik der acentrischen (und somit pyro- und piëzoelectrisch erregbaren) Krystalle zum Gegenstand hat, die Möglichkeit wahrer Pyroelectricität durchweg berücksichtigt.

Bei der principiellen Bedeutung der Frage, ob diese wahre Pyroelectricität in der Wirklichkeit rorkommt, hielt ich es für richtig, mich bei der ersten, immerhin mehr beiläufig vorgenommenen Prüfung nicht zu beruhigen, sondern zu versuchen, eine zweite mit womöglich grösserer Schärfe durchzuführen. Zur Erreichung dieses Zieles war es nach dem Gesagten in erster Linie nothwendig, alle Beobachtungen auf denselben Krystall zu beziehen. Ausserdem habe ich darnach gestrebt, der Bestimmung der pyroelectrischen Constanten des untersuchten Individuums eine grössere Genauigkeit zu geben, als sie Riecke insbesondere deshalb zu erreichen vermochte, weil er Bedenken trug, die benutzten, immerhin ziemlich kostbaren Turmaline zu zerstören, und sie in der natürlichen, an sich für die Beobachtung ungünstigen und überdies nur ungenau auszumessenden Gestalt benutzte. Ich war im Besitze eines für die vorliegenden $Z$ wecke viel geeigneteren Materiales; denn durch die Unterstützung der Kgl. Gesellschaft der Wissenschaften hatte ich seinerzeit für die Elasticitätsuntersuchungen am brasilianischen Turmalin einen prachtvollen Krystall dieses Minerals ankaufen und zu Stäben verarbeiten können; von

1) Vgl. W. Voigt, Wied. Ann. 55. p. 701. 1895. 
letzteren boten aber die parallel der Hauptaxe geschliffenen die denkbar günstigsten Objecte tür die neuen Beobachtungen.

Da für deu beschriebenen Krystall sowohl die Elasticitätsconstanten, bez. -moduln, als auch die piëzoelectrischen Constanten, bez. Moduln bereits gemessen waren, so blieb nur noch die Bestimmung der ihm zugehörigen Constanten der thermischen Dilatation und der Pyroelectricität. Hierüber wird im Folgenden hauptsächlich zu berichten sein.

\section{Die Fundamentalformeln.}

Die Grundlage der von mir aufgestellten allgemeinen Theorie der Piëzoelectricität bildet - wie schon oben gestreift wurde - die Annahme, dass die dielectrischen Momente $a, b, c$ der Volumeneinheit nach den Coordinatenaxen lineare Functionen der Deformationsgrössen $x_{x}, y_{y}, z_{z}, y_{z}, z_{x}, x_{y}$ sind, d. h., dass man setzen kann:

$$
a=\varepsilon_{11} x_{x}+\varepsilon_{12} y_{y}+\varepsilon_{13} z_{z}+\varepsilon_{14} y_{z}+\varepsilon_{15} z_{x}+\varepsilon_{16} x_{y}
$$

hierin stellen die $\varepsilon_{h k}$ die achtzehn, im allgemeinen voneinander unabhängigen piëzoelectrischen Constanten der Substanz dar.

Solange es sich um Deformationen bei constant erhaltenen Temperaturen handelt, sind die Deformationen homogene lineare Functionen der Druckcomponenten $X_{x}, Y_{y}, Z_{z}, Y_{z}, Z_{x}, X_{y}$, sodass man schreiben kann:

$$
-x_{x}=s_{11} X_{x}+s_{12} Y_{y}+s_{13} Z_{z}+s_{14} Y_{z}+s_{15} Z_{x}+s_{16} X_{y}
$$

hierin bedeuten die $s_{h k}$ die Elasticitätsmoduln der Substanz, und es gilt $s_{h k}=s_{k h}$.

Mit Hülfe dieser Beziehungen kann man die dielectrischen Momente auch als lineare Functionen der Drucke darstellen und schreiben:

(3) $-a=\delta_{11} X_{x}+\delta_{12} Y_{y}+\delta_{13} Z_{z}+\delta_{14} Y_{z}+\delta_{15} Z_{x}+\delta_{16} X_{y}$,

wo nun die piezzoelectrischen Moduln $\delta_{h k}$ definirt sind durch die Formeln:

$$
\delta_{i h}=\sum_{k} \varepsilon_{i k} s_{l k k} .
$$


Für die Krystalle der hemimorph-hemiedrischen Gruppe des rhomboedrischen Systemes, zu denen Turmalin zählt, nehmen die vorstehenden Gleichungen die folgenden einfacheren Formen an, bei denen die Z-Axe mit der dreizähligen Hauptaxe, die $X$-Axe mit einer der zweizähligen Nebenaxen zusammenfallend, und die $+Y$-Axe aus einer der die $+Z$-Axe umgebenden Rhomboederflächen $R$ austretend gedacht ist.

Die Momente als Functionen der Deformationsgrössen werden gegeben durch:

$$
\left\{\begin{array}{c}
a=\varepsilon_{15} z_{x}-\varepsilon_{22} x_{y}, \quad b=-\varepsilon_{22}\left(x_{x}-y_{y}\right)+\varepsilon_{15} y_{z}, \\
c=\varepsilon_{31}\left(x_{x}+y_{y}\right)+\varepsilon_{33} z_{z} .
\end{array}\right.
$$

Die Deformationsgrössen als Functionen der Drucke stellen sich dar:

$$
\left\{\begin{array}{l}
-x_{x}=s_{11} X_{x}+s_{12} Y_{y}+s_{13} Z_{z}+s_{14} Y_{z}, \\
-y_{y}=s_{12} X_{x}+s_{11} Y_{y}+s_{13} Z_{z}-s_{14} Y_{z}, \\
-z_{z}=s_{13} X_{x}+s_{13} Y_{y}+s_{33} Z_{z} \\
-y_{z}=s_{14} X_{x}-s_{14} Y_{y} \quad+s_{44} Y_{z}, \\
-z_{x}=s_{44} Z_{x}+2 s_{14} X_{y},-x_{y}=2 s_{14} Z_{x}+2\left(s_{11}-s_{12}\right) X_{y}
\end{array}\right.
$$

Die Beziehungen zwischen den Momenten und den Drucken lauten:

$$
\left\{\begin{array}{c}
-a=\delta_{15} Z_{x}-2 \delta_{22} X_{y},-b=-\delta_{22}\left(X_{x}-Y_{y}\right)+\delta_{15} Y_{z} \\
-c=\delta_{31}\left(X_{x}+Y_{y}\right)+\delta_{33} Z_{z}
\end{array}\right.
$$

wobei nun ist:

$$
\begin{cases}\delta_{22}=\varepsilon_{22}\left(s_{11}-s_{12}\right)-\varepsilon_{15} s_{14}, & \delta_{15}=\varepsilon_{15} s_{44}-2 \varepsilon_{22} s_{14} \\ \delta_{31}=\varepsilon_{31}\left(s_{11}+s_{12}\right)+\varepsilon_{33} s_{13}, & \delta_{33}=2 \varepsilon_{31} s_{31}+\varepsilon_{33} s_{33} .\end{cases}
$$

Aus diesen letzten Formeln erhält man für die piëzoelectrischen Constanten $\varepsilon_{h k}$ die folgenden Ausdrücke in den beiden Gattungen von Moduln:

$$
\begin{cases}\varepsilon_{22}=\frac{\delta_{22} s_{44}+\delta_{15} s_{14}}{s_{44}\left(s_{11}-s_{12}\right)-2 s_{14}^{2}}, & \varepsilon_{15}=\frac{2 \delta_{92} s_{14}+\delta_{15}\left(s_{11}-s_{12}\right)}{s_{44}\left(s_{11}-s_{12}\right)-2 \varepsilon_{14}^{2}}, \\ \varepsilon_{31}=\frac{\delta_{31} s_{33}-\delta_{33} s_{13}}{s_{33}\left(s_{11}+s_{12}\right)-2 s_{13}^{2}}, & \varepsilon_{33}=\frac{-2 \delta_{31} s_{13}+\delta_{33}\left(s_{11}+s_{19}\right)}{s_{33}\left(s_{11}+s_{12}\right)-2 s_{13}^{2}} .\end{cases}
$$


Die thermischen Deformationen können bei mässigen Temperaturänderungen $\boldsymbol{\tau}$ diesen proportional angenommen werden; d. h., man kann setzen:

$$
x_{z}=\alpha_{1} \tau, y_{y}=\alpha_{2} \tau, z_{z}=\alpha_{3} \tau, y_{z}=\alpha_{4} \tau, z_{z}=\alpha_{5} \tau, x_{y}=\alpha_{0} \tau,
$$

wobei die $\alpha_{h}$ die thermischen Deformationscoefficienten darstellon. Für die Krystallgruppe, der der Turmalin zugehört, reducirt sich dies System auf:

$$
x_{x}=y_{y}=\alpha_{1} \tau, \quad z_{z}=\alpha_{3} \tau, \quad y_{z}=0, \quad z_{x}=0, \quad x_{y}=0 .
$$

Demgemäss ist die piëzoelectrische Erregung, welche die Erwärmung eines Turmalines um $\tau$ Grad bei ungeändertem Drucke ergiebt, bestimmt durch:

$$
\left\{\begin{array}{c}
a=0, \quad b=0, \\
c=\frac{2\left(\delta_{31} s_{33}-\delta_{39} s_{13}\right) \alpha_{1}-\left(2 \delta_{31} s_{13}-\delta_{39}\left(s_{11}+s_{12}\right)\right) \alpha_{3}}{s_{33}\left(s_{11}+s_{12}\right)-2 s_{13}^{2}} \tau=\delta \tau,
\end{array}\right.
$$

worin $\delta$ als der Modul der falschen pyroelectrischen Erregung bezeichnet werden kann.

Die gesammte beobachtete Erregung tindet sich innerhalb mässiger Temperaturgrenzen gleichfalls der Erwärmung proportional; es kann also für sie geschrieben werden:

$$
(a)=0,(b)=0, \quad(c)=\gamma \tau,
$$

wobei nun $\gamma$ als Modul der gesammten pyroelectrischen Wirkung erscheint. Die Differenz $(c)-c=c^{\prime}$ stellt dann die nicht auf Deformationswirkungen zurückführbare electrische Erregung dar, und

$$
\gamma-\delta=9
$$

ist somit der Modul der wahren pyroelectrischen Erregung.

Die Frage nach der Existenz wahrer Pyroelectricität im Thrmalin spitzt sich sonach auf die Untersuchung $z u$, ob das so definirte $\vartheta$ einen neben $\gamma$ bez. $\delta$ merklichen Werth besitzt.

Der Plan für ihre Durchführung weicht von dem früher von Riecke und mir verfolgten nur wenig ab; er geht kurz gesagt dahin, dass aus früheren Beobachtungen die Werthe der Elasticitätsmoduln, sowie das Verhältniss der piëzoelectrischen Moduln $\delta_{31} / \delta_{33}$ entnommen werden, und dass an dem früber benutzten Krystall neue Messungen über die thermischen 
Dilatationscoefficienten $\alpha_{1}$ und $\alpha_{3}$ und über das Verhältniss der beiden Moduln $\delta_{33}$ und $\gamma$ angestellt werden.

Der Modul $\delta_{33}$ ist deshalb ausgewählt, weil eine der energischsten piëzoelectrischen Wirkungen, nämlich die Erregung eines zur Hauptaxe parallelen Cylinders durch longitudinalen Druck, ganz allein durch ihn gemessen wird. In der That, setzt man in den Formeln (7)

$$
X_{x}=Y_{y}=Y_{z}=Z_{x}=X_{y}=0, \quad Z_{z}=p,
$$

so resultirt

$$
a=0, \quad b=0, \quad-c=\delta_{33} p .
$$

Bezeichnet dann $\mu$ eine mit $\delta_{33}, \nu$ eine mit $\gamma$ proportionale Grösse, so ist $\gamma-\delta$ proportional mit

$$
\nu-\mu \frac{\left(\left(s_{11}+s_{12}\right) \alpha_{3}-2 s_{13} \alpha_{1}+\left(s_{33} \alpha_{1}-s_{13} \alpha_{3}\right) 2 \delta_{31} / \delta_{33}\right)}{s_{33}\left(s_{11}+s_{12}\right)-2 s_{13}^{2}}=\lambda
$$

und es ist wahre Pyroelectricität vorhanden oder nicht vorhanden, je nachdem $\lambda$ von Null verschieden oder gleich Null ist. Im ersteren Falle ergiebt dann $\lambda / \nu$ den Bruchtheil der ganzen pyroelectrischen Erregung, der als wahre Pyroelectricität $\mathrm{zu}$ bezeichnen ist.

\section{Die elastischen und die piëzoelectrischen Moduln.}

Die Elasticitätsmoduln des benutzten Turmalines habe ich seinerzeit bestimmt ${ }^{1}$ ) und bin dabei zu folgenden Resultaten gekommen, die als Druckeinheit das Kilogrammgewicht pro Quadratcentimeter voraussetzen:

$$
\begin{aligned}
& s_{11}=3,91 \cdot 10^{-7}, s_{12}=-1,01 \cdot 10^{-7} \\
& s_{33}=6,12 \cdot 10^{-7}, s_{13}=-0,16 \cdot 10^{-7} \\
& s_{44}=14,84 \cdot 10^{-7}, s_{14}=+0,57 \cdot 10^{-7} .
\end{aligned}
$$

Beobachtungen über die piëzoelectrischen Moduln sind, wie schon oben gesagt, von Riecke und $\operatorname{mir}^{2}$ ) angestellt und veröffentlicht; indessen ist die Berechnung der Resultate aus den direct gemessenen Werthen früher nicht nach der Methode der kleinsten Quadrate durchgeführt, sondern es ist der Ausgleich der Fehler ziemlich willkürlich vorgenommen worden.

1) W. Voigt, Wied. Ann. 41. p. 712. 1890.

2) E. Riecke u. W. Voigt, Wied. Ann. 45. p. 523. 1892. 
Da es sich bei dem vorliegenden Problem darum handelt, jeder der benutzten numerischen Grundlagen eine möglichst grosse Sicherheit zu geben, so habe ich jene Berechnung nunmehr nachträglich angestellt.

Bezeichnet man die Werthe der in willkürlichen Einheiten ausgedrückten piëzoelectrischen Moduln mit $d_{h k}$, während man unter $\delta_{h k}$ die in absoluten Einheiten ausgedrückten versteht, so sind früher folgende Gleichungen als Ausdruck der directen Messungsresultate aufgestellt ${ }^{1}$ ), bei denen die eingeklammerten Ziffern je die Anzahl der Beobachtungen angeben, von welcher der angegebene Zahlwerth das Mittel darstellt.

$$
\begin{array}{rlr}
\text { beob. } & & \text { ber. } \\
-d_{33}=0,172, & \text { (4) } & 0,171 \\
-d_{22}=0,020, & \text { (2) } & 0,020 \\
-d_{15}-d_{33}-d_{31}-d_{22}=0,500, & \text { (2) } & 0,499 \\
-d_{15}-d_{33}-d_{31}+d_{22}=0,539, & \text { (3) } & 0,540 \\
-d_{31}=0,027, & \text { (1) } & 0,022 \\
-d_{31}=0,025, & \text { (1) } & 0,022 \\
-d_{15}+d_{22}+d_{33}+d_{31}=0,156, & \text { (2) } & 0,154 \\
-d_{15}-d_{22}+d_{33}+d_{31}=0,111, & \text { (1) } & 0,113 .
\end{array}
$$

Giebt man diesen Formeln Gewichte, die den Beobachtungszahlen entsprechen, so erhält man folgendes Werthsystem

$d_{22}=+0,02034, d_{15}=-0,3265, d_{31}=-0,0220, d_{33}=-0,1711 ;$

sein Einsetzen in die links stehenden Ausdrücke liefert die unter „ber." aufgeführten Zahlen. Die Uebereinstimmung ist mit einziger Ausnahme der in fünfter und sechster Reihe stehenden Zahlen, die aus sehr schwachen Erregungen und daher wahrscheinlich ungenauer bestimmt sind, eine überraschend gute.

Für das uns zunächst interessirende Verhältniss $d_{31} / d_{33}$, für das übrigens eine grosse Genauigkeit nicht erfordert wird, ergiebt sich

$$
d_{31} / d_{33}=\delta_{31} / \delta_{33}=0,129 .
$$

Es mag bemerkt werden, dass die oben für die $d_{h k}$ angegebenen Zahlen jedenfalls ein wenig zu klein sind, da sie durch eine Berechnung erhalten wurden, bei der die Capacität

1) E. Riecke u. W. Voigt, 1. c. p. 543 . 
der metallischen Belegungen des gepressten Krystalles gegenüber derjenigen des Electrometers inclusive der Zuleitungen vernachlässigt sind. Die vollständige Theorie der benutzten Messungsmethode habe ich für einen besonders einfachen Fall nachträglich entwickelt. ${ }^{1}$ ) Aus derselben lässt sich schliessen, dass unter den bei unseren Beobachtungen vorliegenden Umständen der gemachte Fehler 1 Proc. jedenfalls nicht erreicht.

Wegen gewisser Anwendungen mögen beiläufig auch diejenigen absoluten Werthe $\delta_{h k}^{\prime}$ der piëzoelectrischen Moduln mitgetheilt werden, die sich bei Einführung des absoluten electrostatischen Maasses ergeben, während der Druck aus praktischen Gründen (wie bisher) in Kilogrammgewicht pro Quadratcentimeter ausgedrückt bleibt. Man hat dann nach dem früher

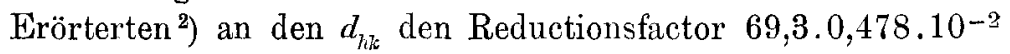
anzubringen und erhält dadurch

$$
\begin{aligned}
\delta_{22}^{\prime}=+0,00674, \delta_{15}^{\prime} & =-0,1081, \delta_{31}^{\prime}=-0,00730 \\
\delta_{33}^{\prime} & =-0,0567 .
\end{aligned}
$$

Will man schliesslich noch als Krafteinheit statt des Kilogrammgewichtes die Dyne einführen, so hat man diese Zahlen mit $9,81.10^{5} \mathrm{zu}$ dividiren; hierdurch findet man

$$
\begin{aligned}
\delta_{22}=+0,687 \cdot 10^{-8}, \delta_{15} & =-11,03 \cdot 10^{-8}, \delta_{31}=-0,744 \cdot 10^{-8}, \\
\delta_{33} & =-5,77 \cdot 10^{-8}
\end{aligned}
$$

als die definitiven Werthe der piëzoelectrischen Moduln des Turmalines in absolutem Maasse (C.G.S.).

\section{Die Constanten der thermischen Deformation.}

Da das hiesige Institut die Hülfsmittel zu so genauen Bestimmungen der thermischen Deformationsconstanten, wie sie für meine $Z$ wecke erwünscht waren, nicht besitzt, so wandte ich mich an die Firma Carl Zeiss in Jena, die das treffliche Fizeau-Abbésche Dilatometer wiederholt ausgeführt und angewandt hat, mit der Bitte, mir ihre Hülfe zu gewähren. Die Geschäftsleitung der Firma hat in entgegenkommendster Weise die nöthigen Beobachtungen mit dem ihr gehörigen Apparat

1) W. Voigt, Wied. Ann. 55. p. 717. 1895.

2) E. Riecke u. W. Voigt l. c. p. 549 . 
durch einen ihrer Mitarbeiter, Hrn. Kellner, ausführen lassen und mir die Resultate zur Verfügung gestellt. Ich spreche auch an dieser Stelle der Firma, wie dem gefälligen Beobachter, nicht minder Hrn. C. Pulfrich, der die bezüglichen Arbeiten geleitet und die Resultate berechnet hat, für die meinen Untersuchungen gewährte Förderung den herzlichsten Dank aus.

Im Nachstehenden wiederhole ich die Angaben, die mir Hr. Pulfrich über die Methode und die Resultate der Beobachtungen gemacht hat.

Aus einem Stück des von mir zu den übrigen Messungen benutzten Turmalines wurde ein Würfel von nahezu $1 \mathrm{~cm}$ Seitenlänge hergestellt, mit dem einen Flächenpaar normal, mit den beiden anderen parallel zu der krystallographischen Hauptaxe orientirt. Die eine der ersteren und die eine der letzteren Flächen wurde darnach soweit hohlgeschliffen, dass von der ursprünglichen ebenen Begrenzung nur noch drei kleine Randstücke übrig blieben, auf denen nun der Würfel, wie auf Füsschen, sicher ruhen konnte. Die diesen umgestalteten Flächen gegenüberliegenden Ebenen wurden sorgfältig polirt.

Als Vergleichskörper diente der Quarzring von ca. $1,01 \mathrm{~cm}$ Höhe, dessen axiale thermische Dilatation Hr. E. Reimerdes ${ }^{1}$ ) sorgfältig bestimmt hat. Das Turmalinpräparat wurde mit dem Ringe, und zwar in dessen Innenraum, auf eine ebene Grundplatte von Quarz gestellt und auf den Ring eine plane Deckplatte von Quarz aufgelegt. In dem Raum zwischen der oberen Fläche des Turmalines und der unteren der Deckplatte kommen dann bei geeigneter Beleuchtung die bekannten, als Beobachtungsobject dienenden Interferenzstreifen zu Stande. Bezüglich des Details der Beobachtungsmethode und der zur Berechnung anzuwendenden Formeln kann auf die Arbeit des Hrn. Reimerdes verwiesen werden. Es genügt hier Folgendes hervorzuheben.

Die Lagenbestimmung der Interferenzstreifen für die rothe Wasserstofflinie und die grüne Quecksilberlinie erfolgte durch mikrometrische Ausmessung mit Hülfe des Dilatometers bei Temperaturen von ca. 10,60, $90^{\circ} \mathrm{C}$, und nach der Abkühlung wieder bei ungefähr $10^{\circ} \mathrm{C}$. Aus der ersten, zweiten und

1) E. Reimerdes, Inang.-Diss., Jena 1896.

Ann. đ. Phys, u. Chem. N. F. 66. 
vierten Beobachtung wurde die Zahl der "verschobenen Streifen sowohl für die Erwärmung, als für die Abkühlung ermittelt und hieraus der Ausdehnungscoefficient für eine mittlere Temperatur von ca. $35^{\circ}$ abgeleitet. Die Combination der ersten, dritten und vierten Beobachtung ergab den Ausdehnungscoefficienten für eine mittlere Temperatur von ungefähr $50^{\circ}$. Die aus Erwärmung und Abkühlung für dieselbe mittlere Temperatur gewonnenen Ausdehnungscoefficienten stimmten bis auf weniger als 1 Proc. untereinander überein.

Unter Benutzung des von Hrn. Reimerdes für den Quarzring gefundenen axialen Ausdehnungscoefficienten

$$
\alpha_{q}=(6,925+0,01689 t) 10^{-6},
$$

in dem $t$ die Temperatur nach Celsius bezeichnet, ergaben die mittleren Beobachtungsresultate folgende Zahlen:

1. Turmalin, Ausdehnung normal zur Hauptaxe.

Dicke des Präparates $0,9686 \mathrm{~cm}$, mittlere Temperatur $41,0^{0} \quad \alpha_{1}=3,587.10^{-6}$,

Hieraus

$$
52, \tilde{0}^{0} \quad \alpha_{1}=3,729 \cdot 10^{-6} \text {. }
$$

$$
\alpha_{1}=(3,081+0,01235 t) 10^{-6}
$$

2. Turmalin, Ausdehnung parallel zur Hauptaxe. Dicke des Präparates $0,9900 \mathrm{~cm}$,

- mittlere Temperatur $\begin{aligned} 33,5^{0} & \omega_{3}=8,530 \cdot 10^{-6}, \\ 50,5^{0} & \alpha_{3}=8,895 \cdot 10^{-6}\end{aligned}$

Hieraus

$$
" \quad \quad \quad \quad 50,5^{0} \quad \alpha_{3}=8,895 \cdot 10^{-6} \text {. }
$$

$$
c_{3}=(7,810+0,0215 t) \cdot 10^{-6} .
$$

Diese Werthe stimmen befriedigend mit den von Fizeau an grünem brasilianischen Turmalin erhaltenen überein, welche lauten

$$
\alpha_{1}=(3,06+0,0183 t) \cdot 10^{-6}, \quad \alpha_{3}=(7,77+0,0320 t) \cdot 10^{-6} .
$$

Dagegen weichen sie stark von den von Riecke und mir früher benutzten Pfaff'schen Zahlen

$$
\alpha_{1}=7,73 \cdot 10^{-6}, \quad \alpha_{3}=9,37 \cdot 10^{-6}
$$

$a b$, deren Unterschied von den Fizeau'schen uns seinerzeit nicht aufgefallen ist, und es steht demgemäss von vornherein zu erwarten, dass die neue Untersuchung das von uns früher erhaltene Resultat nicht bestätigen wird. 


\section{Das Verhältniss der pyroelectrischen Constante $\gamma$ des Turmalines zu seinem piëzoelectrischen Modul $\delta_{33}$.}

Wie schon auf p. 1037 erörtert ist, bedarf man zur Entscheidung der Frage nach der Existenz wahrer Pyroelectricität beim Turmalin nicht des absoluten Werthes seiner pyroelectrischen Constante $\gamma$, sondern nur deren Verhältniss zu einem seiner piëzoelectrischen Moduln, z. B. zu $\delta_{33}$. Dies lat für unsere Arbeit den Vortheil, dass für unsere nächsten Zwecke jene Hülfsbeobachtungen, die nöthig sind, um das benutzte Messinstrument - hier ein Thomson'sches Electrometer - zur absoluten Messung von Ladungen zu aichen, unterbleiben konnten.

Der Grundgedanke der zur Bestimmung des Verhältnisses $\gamma / \delta_{33}$ angewandten Beobachtungsmethode war kurz der, dass mit den Quadrantenpaaren des Electrometers in paralleler Schaltung verbunden war sowohl das Krystallpräparat $k_{1}$, an dem die pyro-, als $k_{2}$, an dem die piëzoelectrische Erregung beobachtet werden sollte, und dass abwechselnd das eine oder das andere erregt und dabei das Electrometer abgelesen

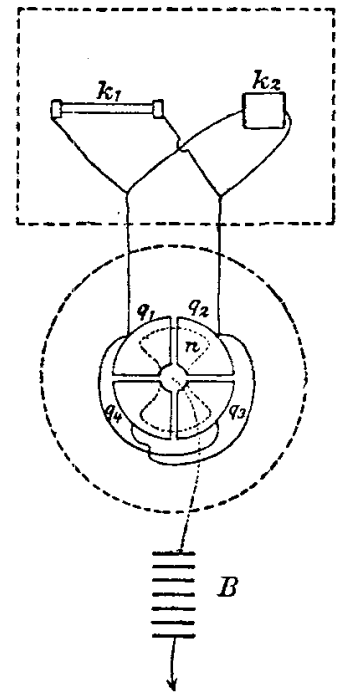

Fig. 1. wurde. Da bei diesen Beobachtungen die mit den Quadrantenpaaren verbundenen Capacitäten unverändert die gleichen waren, so entsprachen direct gleiche Ausschläge des Electrometer's gleichen frei werdenden Ladungen; da weiter bei der pyroelectrischen Erregung die Temperatur-, bei der piëzoelectrischen die Druck-Aenderung verfügbar war, so konnten diese Ausschläge einander so nahe gleich gemacht werden, dass es nicht einmal nöthig wurde, die Proportionalität der Ausschläge mit den Ladungen zu prüfen.

Die obenstehende Fig. 1 erläutert schematisch die Anordnung. $q_{1}$ bis $q_{4}$ sind die diagonal gekoppelten Quadranten des Electrometers; mit jedem Paar ist je eine Belegung des 
$z u$ erwärmenden Krystallstäbchens $k_{1}$ und eine des der zu pressenden Prismas $k_{2}$ verbunden. Alle Verbindungen sind durch Löthung hergestellt, um eine Veränderung der Contacte möglichst auszuschliessen. Die Nadel des Electrometers war an einem Wollaston-Platindraht aufgehängt und durch denselben mit dem einen Pol einer sorgsam isolirten Wasserbatterie $B$ verbunden, deren anderer Pol zur Erde abgeleitet wurde.

Die Krystallpräparate $k_{1}$ und $k_{2}$ mit den nöthigen Hülfsmitteln zu ihrer thermischen und mechanischen Erregung befanden sich in einem Glaskasten, der mit Drahtgitter, bez. Stanniol überzogen war, um jene Theile der Wirkung von störenden thermischen und electrischen Einflüssen nach Möglichkeit zu entziehen. Wir wollen diesen Kasten weiterhin kurz den lsolirkasten nennen.

Das Electrometer war mit einem ähnlichen Gehäuse umgeben; zwischen beiden blieb nur ein geringer Raum frei, der eben genügte, um die ihn durchsetzenden Leitungsdrähte, und damit die Electrometerquadranten bequem zur Erde abzuleiten, sowie solches nöthig wurde.

Die beiden Schutzhüllen sind in der obigen schematischen Figur durch punktirte Linien angedeutet.

Ueber die Einrichtung des Electrometers ist sonst nichts zu sagen, als dass gemäss der ihm gestellten Aufgabe, Ladungsstärken zu vergleichen, seine Isolation durch ausschliessliche Verwendung von Schellackstiutzen und -trägern so sehr als möglich vergrössert und seine Capacität durch Auseinanderschieben der Quadranten verkleinert war. Seine Empfindlichkeit war derart, dass ein Cadmiumelement von 1,024 Volt Spannung bei Commutation auf der Scala einen Ausschlag von $90 \mathrm{~mm}$ gab. -

Der zu pressende Krystall $k_{2}$ hatte die Gestalt eines rechtwinkeligen Parallelepipeds und war in der früher beschriebenen Weise ${ }^{1}$ ) an zwei gegenüberliegenden Flächen mit Stanniol überzogen, dagegen auf den übrigen zur Erhöhung der Isolation lackirt. Er befand sich mit den Belegen zwischen zwei kleinen ebenen Kupferplatten, deren eine, auf einem

1) E. Riecke u. W. Voigt, l. c. p. 530. 
Schellackklötzchen befestigte, seine Unterlage bildete, während auf die andere, ebenfalls mit Schellack nach aussen isolirte, das Gewicht wirkte, das den Druck auf den Krystall hervorbringen sollte. Es wurden die beiden früher mit $A$ und $B$ bezeichneten Turmalinpräparate benutzt, die eine Kante der krystallographischen Hauptaxe parallel hatten und nach der Richtung dieser Kante comprimirt wurden. $A$ hatte erheblich grössere Querdimensionen als $B$, und dies liess $A$ zu den Hauptbeobachtungen weniger geeignet erscheinen. Da nämlich die Zimmertemperatur sich nicht vollständig constant erhalten liess, so variirten auch die zu piëzoelectrischen Untersuchungen benutzten Präparate dauernd ein wenig ihre Temperatur; sie wurden also fortwährend auch pyroelectrisch erregt. Die in ihren Belegungen frei werdenden Ladungen sind nun aber bei gleichen Temperaturänderungen den Querschnitten der Präparate proportional; sie waren sonach bei dem grösseren Präparat erheblich stärker, als bei dem kleineren. Wenn nun auch unter Voraussetzung einer constanten Geschwindigkeit der Temperaturänderung diese pyroelectrische Erregung aus dem Mittelwerth der bei Belastung und der bei Entlastung gewonnenen Beobachtungen streng heruusfällt, so konnte doch bei dem grösseren Präparat eine Abweichung von der constanten Geschwindigkeit recht wohl einen merklichen Fehler geben, und deshalb ist der grössere Krystall $A$ nur insoweit benutzt, als constatirt wurde, dass er merklich dieselbe piëzoelectrische Constante besass, als der kleinere $B$. Bei diesem übertrafen die pyroelectrischen Erregungen nicht 2 Proc. der piëzoelectrischen, sie waren aber meist noch geringer.

Ueber die zur Messung der piëzoelectrischen Erregung zu machenden Ablesungen ist in der früheren Arbeit ausführlich gesprochen. Hier genügt die Erwähnung, dass zur Elimination der Wirkung einer nicht vollkommenen Isolation die auf Belastung oder Entlastung folgenden fünf Umkehrpunkte der Electrometernadel beobachtet wurden.

Die einer wechselnden Temperatur auszusetzenden Turmalinstäbchen $k_{1}$ waren gleichfalls oberflächlich leicht lackiert. Die leitenden Belegungen ihrer Enden wurden durch zwei kleine, aus Kupferfolie gefertigte Kästchen $h_{1}, h_{2}$ (Fig. 2) gebildet, die über diese Enden geschoben waren und zugleich 
die Stäbchen trugen. Zu letzterem Zweck waren sie mit einander zugewandten Oeffnungen an die Enden zweier dünner kupferner Federn $f_{1}, f_{2}$ befestigt, die ihrerseits an einem Schellackstab $s$ sassen; der Stab seinerseits war an das untere Ende eines dünnen Glasstabes $g$ gekittet, welcher die Stütze des ganzen Systemes bildete. Dieser Glasstab hing bei den Beobachtungen von der Decke des oben erwähnten Isolirkastens herab, sodass das untere Ende mit dem daran befestigten Krystallstäbchen pendelnde Bewegungen ausführen konnte. An

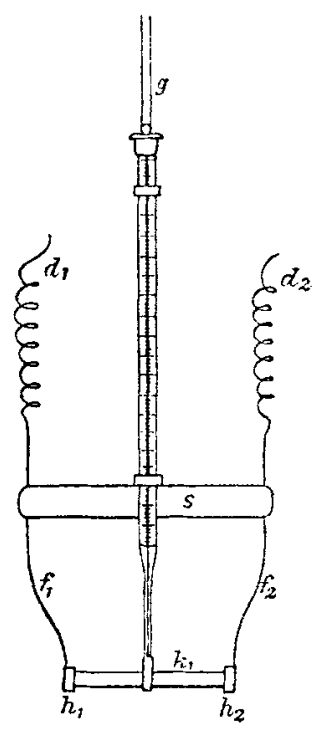

Fig. 2. die Federn $f_{1}^{\prime}, f_{2}$ waren die Drähte $d_{1}, d_{2}$ angelöthet, die zu den Quadranten des Electrometers führten.

Der Glasstab trug ausser dem Krystallpräparat noch ein empfindliches Thermometer, dessen Gefüss dicht an dem Krystall anlag; zwei in angemessener Stellung an dem Thermometer befestigte Lupengläser gestatteten, von ausserhalb des Isolirkastens her HundertelGrade abzulesen.

Unter dem Stäbchen stand ein primitiver niedriger Wagen, der sich von ausserhalb des Isolirkastens hin- und herschieben liess. Er trug zwei Gefässe mit Paraffinöl, die durch Umkleidung mit Filz gegen Wärmeaufnahme und -abgabe einigermaassen geschützt waren. Das eine wurde vor Beginn der Beobachtung um $2-3^{\circ} \mathrm{C}$. über, das andere um ebensoviel znter die Temperatur der Umgebung gebracht. Diese Temperaturen änderten sich dann nur ausserordentlich langsam, und es erwies sich vortheilhafter, bei allmählich abnehmender Temperaturdifferenz zu beobachten, als letztere durch eigene Veranstaltungen, welche leicht die electrischen Beobachtungen stören, vollständiger constant zu halten. Die eigentliche Messung geschah dann in folgender Weise.

Während die Quadranten des Electrometers zur Erde abgeleitet waren, wurde das eine der beiden Oelgefässe unter das oben beschriebene, Krystall und Thermometer tragende 
System geschoben und letzteres bis nahe an den Schellackstab eingetaucht. Mit Hülfe eines von aussen in den Isolirkasten geleiteten Fadens wurde dann der untere Theil jenes Systemes in pendelnde Bewegungen versetzt, sodass das Oelbad ziemlich kräftig umgerührt und zugleich der Temperaturausgleich zwischen dem Bad einer-, dem Stäbchen und dem Thermometer andererseits beschleunigt wurde.

War keine Temperaturänderung an dem Thermometer mehr merklich, so wurde die Verbindung der Quadranten mit der Erde aufgehoben und die Ablesung von drei Umkehrpunkten der Nadel ausgeführt. Hierauf wurde Stäbchen und Thermometer aus dem ersten Oelbad gehoben, das andere Bad untergeschoben, das ganze System wie zuvor eingetaucht und in Schwingungen versetzt. Schon nach einer Periode $2 T$ der Electrometernadel (ca. 25 Secunden) pflegte eine Aenderung des Standes des Thermometers nicht mehr merklich zu sein, und da das Krystallstäbchen erheblich dünner war, als das Thermometergefäss, so durfte man zu diesem Zeitpunkt den Ausgleich seiner Temperatur mit der des Bades als innerhalb der Genauigkeit der Ablesung am Thermometer vollzogen betrachten.

Die Ablesungen an der Electrometernadel begannen bei deren dritter Umkehr, also um etwa $5 / 2 T$ nach dem Moment des Eintauchens; sie wurden bis zur siebenten Umkehr fortgesetzt. Aus den so erhaltenen fünf Zahlen lässt sich dann, wie weiter unten gezeigt werden wird, die Ablenkung berechnen, welche die Nadel erlitten haben würde, wenn das Stäbchen die Temperatur des zweiten Bades momentan erreicht bätte, oder wenn die Isolation eine vollständige gewesen wäre.

Nach Ausführung dieser Ablesungen wurden die Quadranten wieder zur Erde abgeleitet, nach Wiederherstellung der Isolation drei Umkehrpunkte der Nadel bestimmt und darauf der Wechsel der Bäder, wie oben, von neuem ausgeführt. Die Beobachtungen gingen so schnell von statten, dass in der Zeit von einer Benutzung eines jeden Bades bis zur nächsten seine Temperatur selten mehr als $0,1^{\circ} \mathrm{C}$. variirte.

Die für die theoretische Verwerthung bestimmten Beobachtungsreihen sind in nachstehender Tabelle im Auszug mitgetheilt. Die bezüglichen Messungen wurden unmittelbar hinter- 
einander innerhalb weniger Stunden und somit bei möglichst constanten Verhältnissen angestellt, nachdem durch eine grössere Zahl orientirender Versuche jeder Theil der Beobachtungsmethode exprobt worden war. Die mannichfachen Veränderungen, die hierbei die Details der letzteren erlitten, können mit Stillschweigen übergangen werden. Bei den Vorarbeiten, wie bei den definitiven Messungen hat mir der Assistent des Institutes, Hr. Wi echert, vielfältige Hülfe geleistet.

Stäbchen I. $q=0,0555,11$ Beob.

$$
\begin{aligned}
& r_{1}=20,73 \quad 372,7 \quad 470,3 \quad 418,1 \quad y_{1}=436,2 \\
& I_{2}=24,41 \quad 309,2 \quad 324,5 \quad 319,1 \quad 325,2 \quad 324,2 \quad x_{2}=317,4 \\
& \boldsymbol{r}=3,68 \quad \xi=+4,6 \quad y_{1}-x_{2}=118,8 \text {; } \\
& \tau_{2}=24,45 \quad 499,0 \quad 398,7 \quad 445,1 \quad y_{2}=429,3 \\
& \boldsymbol{I}_{1}=20,79 \quad 570,9 \quad 547,7 \quad 556,3 \quad 548,5 \quad 549,8 \quad x_{1}=558,1 . \\
& \tau=3,66 \quad \xi=-3,6 \quad x_{1}-y_{2}=128,8 \text {; }
\end{aligned}
$$

Mittel $\tau=3,67, \sigma_{\tau}=130,0, \sigma_{\tau} / \tau q=638$.

Krystall $B . \quad 6$ Beob.

$$
\begin{aligned}
& \text { R. } 437,7 \quad 426,1 \quad 430,4 \quad y=429,3 \\
& \text { B. } 662,5 \quad 530,6 \quad 595,7 \quad 556,8 \quad 573,6 \quad x=579,7 \\
& y-x=150,4 \\
& \text { R. } 434,7 \quad 427,7 \quad 430,5 \quad y=429,6 \\
& \text { E. } 192,0 \quad 322,4 \quad 255,9 \quad 294,1 \quad 275,6 \quad x=275,2 \\
& x-y=154,4 \\
& \text { Mittel } \sigma_{p}=152,4, \quad \sigma_{y}, P=30,4
\end{aligned}
$$

Stäbchen II. $q=0,0599,10$ Beob.

$$
\begin{aligned}
& \tau_{1}=19,32 \\
& 405,8 \quad 532.4 \quad 465,0 \\
& 313,9 \quad 336,0 \quad 326,5 \quad 333,6 \quad 331,7 \quad x_{1}=326,9 \\
& \tau_{\mathrm{g}}=23,71 \\
& t=4,39 \\
& \xi=+4,0, \quad y_{1}=x_{2}=161,5 \text {; } \\
& \tau_{\mathrm{a}}=23,71 \\
& 558,9 \quad 435,1 \quad 498,9 \\
& y_{2}=477,2 \\
& \tau_{1}=19,39 \\
& 655,4 \quad 628,6 \quad 638,1 \quad 628,3 \quad 629,4 \\
& x_{1}=641,4 \\
& \tau=4,32 \\
& \boldsymbol{\xi}=-6,0, \quad x_{1}-y_{2}=164,2 \text {; } \\
& \text { Mittel } \tau=4,35_{5}, \quad \sigma_{\tau}=170,4 \quad \sigma_{\tau} / \tau q=652 .
\end{aligned}
$$

Krystall $B .6$ Beob.

$$
\begin{aligned}
& \text { R. } 464,0 \quad 460,9 \quad 460,7 \quad y=461,2 \\
& \text { B. } 693,5 \quad 562,3 \quad 626,3 \quad 587,4 \quad 603,8 \quad x=611,5 \\
& x-y=150,3 \\
& \text { R. } 466,8 \quad 458,3 \quad 461,8 \quad y=460,8 \\
& \text { E. } 230,2 \quad 357,9 \quad 292,9 \quad 329,3 \quad 311,5 \quad x=311,9 \\
& y-x=148,9 \\
& \text { Mittel } \sigma_{p}=149,6, \quad \sigma_{p} / P=29,8_{n} \text {. }
\end{aligned}
$$


Stäbchen III. $\quad q=0,0599,10 \mathrm{Beob}$.

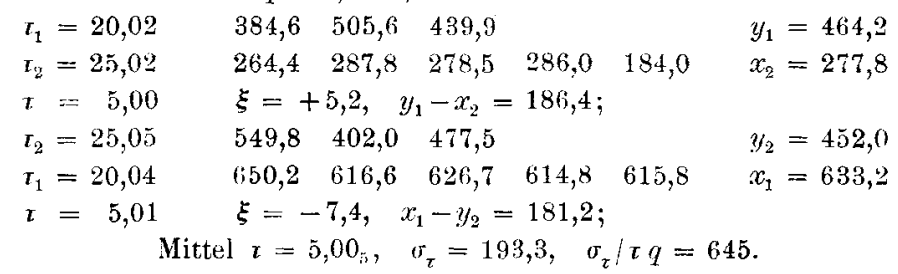

Krystall $B$. $\quad$ S Beob.
R. $451,5 \quad 450,6 \quad 449,7$
$y=450,4$
B. 684,
551,9
616,5
577,2
$593,4 \quad x=600,7$
$x-y=150,3$
R. 457,6
$452,7 \quad 453,3$
$y=453,5$
E. 213,4
344,9
$277,2 \quad 314,2$
$y-x=155,2$
Wittel $\sigma_{p}=152,7, \quad \sigma_{p} / P=40,4$.

Was die Bedeutung der obigen Zahlen angeht, so findet sich neben der Ordnungsnummer des pyroelectrisch erregten Stäbchens zunächst sein Querschnitt q, genauer, das Mittel aus der Grösse seiner beiden Endquerschnitte, in Quadratcentimetern. Daneben steht die Anzahl der überhaupt mit dem Stäbchen ausgeführten Beobachtungsreihen, von denen etwa die Hälfte den Uebergang von niederen zu höheren, die Hälfte den Uebergang von höheren zu niederen Temperaturen betrifft.

Die darnach folgenden Zablen sind die Mittel aus den für diese beiden Erregungen erhaltenen Messungen. $\tau_{1}$ bezeichnet die mittlere Temperatur des kälteren, $\tau_{2}$ die des wärmeren Bades zur Zeit, da Wärmegleichgewicht mit dem Stäbchen herrschte. Neben den Werthen von $\tau_{1}$ und $\tau_{2}$ stehen die Mittelwerthe der bei diesen Temperaturen erhaltenen drei bez. fünf Umkehrpunkte der Electrometernadel auf der Scala.

In Bezug auf die rechnerische Verwerthung dieser Zahlen bemerke ich folgendes.

Wenn die Nadel einem constant wachsenden oder abnehmenden electrischen Einfluss unterliegt, der ihre Ruhelage während einer einfachen Schwingung $T^{\prime}$ um $\xi$ Scalentheile verschiebt, und wenn $d$ ihr Dämpfungsverhältniss bezeichnet, so gilt für die einem Durchgang durch die Ruhelage folgenden Umkehrpunkte $a_{h}$ das Gesetz ${ }^{1}$ )

1) E. Riecke u. W. Voigt, 1. c. p. 033. 


$$
\begin{aligned}
& a_{1}=x+\frac{1}{2} \xi+\iota, \\
& a_{2}=x+\frac{3}{2} \xi-\iota / d, \\
& a_{3}=x+\frac{5}{2} \xi+\kappa / d^{2}, \\
& a_{4}=x+\frac{7}{2} \xi-\alpha / d^{3} . \\
& \iota_{5}=x+\frac{9}{2} \xi+\alpha / d^{4},
\end{aligned}
$$

Hierin bezeichnet $x$ die Ruhelage, die eintreten würde, wenn von dem Zeitpunkt des ersten Durchganges an die electrische Einwirkung auf die Nadel constant bliebe. Das Dämpfungsverhältniss $d$ ist durch die Beobachtung der Schwingungsamplituden bei feststehender Ruhelage, d. h. bei verschwindendem $\xi$, zu 1,88 bestimmt worden; diese Zahl ist bei den weiter unten beschriebenen Rechnungen benutzt worden. Die Verschiebung $\xi$ hat im allgemeinen eine doppelte Ursache. Einerseits ändert sich während der Ablesungen die Temperatur des, wie oben beschrieben, mit dem beobachteten Stäbchen $k_{1}$ parallel geschalteten Krystalles $k_{2}$ ein wenig, andererseits ist die Isolation des ganzen Systemes mangelhaft.

Während sich das Stäbchen im ersten Bad befindet, trägt es nach der obigen Darstellung eine merkliche Ladung nicht, eine Einwirkung der mangelbaften Isolation findet somit nicht statt; $\xi$ beruht hier allein auf der geringen Temperaturänderung in $k_{2}$ und ist demgemäss ziemlich unbedeutend, überdies bei aufeinander folgenden Beobachtungsreihen von gleichem Vorzeichen und von nahe gleicher Grösse. Demgemäss wird es passend durch Combination der aufeinander folgenden $\mathrm{Be}-$ obachtungsreihen, die abwechselnd Erwärmung und Abkühlung benutzen, eliminirt.

Aus den drei auf das erste Bad bezüglichen Ablesungen $a_{1}, a_{2}, a_{3}$ sind die in der Tibelle aufgeführten Grössen $y$ berechnet nach der Formel

$$
y=\frac{a_{1}+2 \alpha_{2} d+a_{3} d^{2}}{(1+d)^{2}}=x+\frac{1+5 d}{2(1+d)} \xi ;
$$

das in ihr neben $x$ stehende Glied fällt bei der definitiven Verwerthung dieser Zahlen nach dem oben Gesagten heraus.

Bei den auf das zweite Bad bezuiglichen Beobachtungen kommen beide Ursachen, welche die Verschiebung $\xi$ bedingen, 
gemeinsam zur Geltung; dabei wirkt die zweite, wesentlichere, bei Erwärmung und bei Abkühlung im entgegengesetzten Sinne; $\boldsymbol{\xi}$ muss daher hier bei jeder dieser Beobachtungen für sich eliminirt werden. Dies geschieht mit Hülfe der fünf angegebenen Ablesungen $a_{1}, \ldots a_{5}$ nach der Formel

$$
x=x_{1}-\left(x_{4}-x_{1}\right)_{6} \frac{1+3 d}{(1+d)},
$$

in der bezeichnet

$$
x_{1}=a_{2}+\frac{a_{1}-a_{2}}{1+\frac{a}{d}}, x_{4}=a_{5}+\frac{a_{4}-a_{5}}{1+d} .
$$

Die Grösse $x$ ist oben in der Tabelle gleichfalls aufgeführt; sie stellt nach dem zuvor Gesagten diejenige Ruhelage dar, die eintreteten würde, wenn von einem Zeitpunkt an, der um $\frac{1}{2} T$ vor der ersten Ablesung liegt, die electrischen Verhältnisse constant blieben. Dieser Zeitpunkt liegt aber um $2 T$ nach dem Moment des Eintauchens des Stäbchens in das zweite Bad, und um die Wirkung der mangelhaften Isolation etc. vollständig zu eliminiren, muss man noch den Vorgang in Rechnung ziehen, der sich während dieser, vor den mitgetheilten Messungen liegenden Zeit abgespielt hat.

Hätte die electrische Erregung des Stäbchens diese ganze Zeit hindurch die volle Stärke besessen, so wäre auch die Verschiebung der Ruhelage mit dem vollen Betrag $\xi$ in Rechnung zu setzen; in diesem Falle wäre der wahre Ausschlag $\sigma_{r}$, der einer pyroelectrischen Erregung durch die Temperaturänderung $\tau=\tau_{2}-\tau_{1}$ entspricht, durch die Formel

$$
\pm \sigma_{r}=y-x+2 \xi
$$

gegeben. Indessen nimmt das Stäbchen nicht momentan die Temperatur des zweiten Bades an, seine Erregung erreicht erst allmählich den vollen Werth; somit ist auch der Verlust, den seine Ladung erleidet, geringer, und man wird zu setzen haben

$$
\pm \sigma_{r}=y-x+2 \beta \xi,
$$

worin $\beta$ einen echten Bruch bezeichnet. Es handelt sich darum, die Grösse von $\beta$ und von $\xi$ zu bestimmen, wobei eine mässige Genauigkeit ausreichen wird, da $2 \beta \xi$ immerhin nur eine Correction ist, die an dem (neben ihr sehr beträchtlichen) Werth von $y-x$ angebracht werden soll. 
$\xi$ habe ich aus den letzten drei der für das zweite Bad mitgetheilten Ablesungen bestimmt, weil bei diesen die Amplituden schon kleiner waren, und darum die strengere Gültigkeit des oben angegebenen Gesetzes erwartet werden durfte. Es gilt, wie leicht erkennbar ist,

$$
\xi=\frac{\left(a_{4}-a_{3}\right) d+\left(a_{5}-a_{4}\right)}{1+d} .
$$

Die nach dieser Formel berechneten Werthe $\xi$ sind in der Tabelle angegeben; sie finden sich bei ein und demselben Stäbchen von etwas abweichender absoluter Grösse, je nachdem dasselbe durch Erwärmung oder durch Abkühlung erregt war. In der That enthält $\xi$ nach dem oben Gesagten einen Theil, der sein Vorzeichen hierbei nicht umkehrt, nämlich die Wirkung der veränderlichen Temperatur des dem Stäbchen parallel geschalteten Krystalles $k_{2}$.

Fasst man die von jenem Antheil freien mittleren absoluten Werthe der gefundenen $\xi$ für die drei beobachteten Stäbchen ins Auge, welche bez. lauten

$$
\xi_{I}=4,1, \quad \xi_{I I}=5,0, \quad \xi_{I I I}=6,3,
$$

und vergleicht damit die mittleren absoluten Werthe $x-y$ oder auch die durch sie angenähert gemessenen electrischen Erregungen $\varepsilon$ der Stäbchen, nämlich die Zahlen

$$
\varepsilon_{I}=124, \varepsilon_{I I}=163, \delta_{I I I}=184 \text {, }
$$

so erhält man

$$
\left(\begin{array}{l}
\varepsilon \\
\xi
\end{array}\right)_{I}=30,\left(\begin{array}{c}
\varepsilon \\
\xi
\end{array}\right)_{I I}=33,\left(\begin{array}{l}
\varepsilon \\
\xi
\end{array}\right)_{I I I}=29 .
$$

$\xi$ ist sonach mit $\varepsilon$ nahe proportional, wie das zu erwarten war, wenn anders die Verschiebung der Ruhelage auf der mangelhaften Isolation beruhte.

Um $\beta$ zu erhalten, musste zunächst der Verlauf der Temperatur und somit der electrischen Erregung des Stäbchens während der ersten Doppelschwingung der Nadel nach dem Eintauchen des Stäbchens in das zweite Bad bestimmt werden. Die Temperatur des Stäbchens durfte in hinreichender Annäherung der des mit ihm verbundenen Thermometergefässes gleichgesetzt werden und liess sich sonach leicht bestimmen. Die in gleichen Zeitintervallen abgelesenen Temperaturen wurden als Ordinaten aufgetragen und mit ihrer Hülfe die Curve 
construirt, welche den zeitlichen Verlauf der Temperatur und somit denjenigen der Erregung des Stäbchens angenähert darstellte (vgl. Fig. 3). Da in jedem Zeitelement das Stäbchen einen Betrag an Ladung verliert, der seiner augenblicklichen kirregung nahezu proportional ist, so verhält sich der wirkliche Verlust innerhalb der ersten Doppelschwingung zu dem in den folgenden stattfindenden, wie die Fläche $a b c d a$ zu dem Rechteck $a b c d^{\prime} a$ in der Figur. Dies Verhältniss, das mit dem oben eingeführten $\beta$ über-

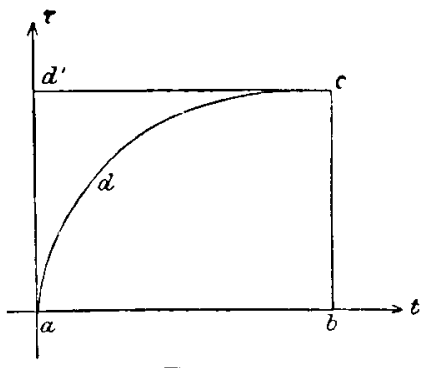

Fig. 3. einstimmt, wurde bestimmt durch Wägung der beiden betreffenden Stücke des Cartonpapiers, auf dem die Construction der Curve vorgenommen war. Sein Werth fand sich sehr genau gegeben durch

$$
\beta=0,75 .
$$

Mit Hülfe der oben mitgetheilten Zahlen für $\xi$ und dieser Zahl für $\beta$ sind die in der Tabelle mitgetheilten Zahlenwerthe für $\sigma_{\tau}$ gemäss der Formel (17) berechnet. Aus ihnen ergiebt sich dann sogleich der Ausschlag, den ein Stäbchen von dem Querschnitt eines Quadratmillimeters bei einer Temperaturänderung von $1^{\circ} \mathrm{C}$. liefern würde, indem man $\sigma_{\tau}$ mit dem angegebenen Querschnitt $q$ und der benutzten mittleren Temperaturänderung $\tau$ dividirt, also bildet

$$
\nu=\sigma_{\tau} / \tau q .
$$

Die erhaltenen Zahlen für $v$, nämlich

$$
\text { I. } 638 \text {, II. } 652 \text {, III. } 645 \text {, }
$$

stimmen so gut überein, als nur irgend zu hoffen war. Ihr Mittel ist

$$
\nu=645,
$$

und mit dieser für die ganze pyroelectrische Erregung des Turmalines charakteristischen Zahl wird weiter unten zu rechnen sein.

Auf die Beobachtungen über die pyroelectrische Erregung eines Turmalinstäbchens folgen in der Tabelle stets die im Anschluss daran erhaltenen Zahlen über die piëzoelectrische 
Erregung desselben Krystallparallelepipedes, das in der früheren Arbeit durch den Buchstaben $B$ bezeichnet war.

Zuerst findet sich wieder die Anzahl der Beobachtungsreihen, aus denen die mitgetheilten mittleren Ablesungen berechnet sind. Daran schliessen sich die beobachteten Umkehrpunkte der Nadel; drei, mit $R$ bezeichnete, sind unmittelbar nach Aufhebung der Ableitung erhalten, welche zuvor die Belegungen des Krystalles mit der Erde verband, und dienen zur Bestimmung der Ruhelage $y$ der Nadel in der oben erörterten Weise; fünf andere beziehen sich auf die einer Belastung $(B)$ oder Entlastung $(E)$ unmittelbar folgenden Umkehrpunkte und sind in der gleichfalls oben besprochenen Weise zur Berechnung der Lagen $x$ benutzt, welche die Nadel ohne Electricitätsverlust eingenommen haben würde. Die Differenz $\pm \sigma=x-y$ giebt hier aber unmittelbar den der Be- oder Entlastung entsprechenden Ausschlag; eine Correction ist nicht anzubringen, weil die Ablesungen direct nach der mechanischen Einwirkung auf den Krystall begannen. Zwar steckt in den so erhaltenen $\sigma$ noch die Wirkung der oben erörterten allmählichen Temperaturänderung des gepressten Krystalles; dieselbe fällt aber aus dem Mittel $\sigma_{p}$ der beiden bei Be- und bei Entlastung erhaltenen Zahlen heraus.

Die Belastung $P$ betrug bei allen Beobachtungen übereinstimmend $5,025 \mathrm{~kg}$. Reducirt man den Ausschlag auf $1 \mathrm{~kg}$, d. h., bildet man

$$
\mu=\sigma_{p} / P
$$

so erhält man die oben angeführten Zahlen, welche bei den drei Beobachtungsgruppen lauten:

Ihr Mittel:
1. 30,4 ,
2. $29,8_{5}$,
3. 30,4 .

$$
\mu=30,2
$$

ist die weiter unten zu benutzende für die axiale piëzoelectrische Erregung charakteristische Zahl.

Dieselbe setzt voraus, dass der Druck in Kilogrammen pro Quadratcentimeter ausgedrückt ist.

\section{Besitzt der Turmalin wahre Pyroelectricität?}

Die Frage nach der Existenz wahrer Pyroelectricität am Turmalin spitzt sich nach dem p. 1038 Gesagten zu auf die 
Untersuchung des Werthes der Grösse $\lambda$, laut (16) gegeben durch:

$$
v-\mu \frac{\left(\left(s_{11}+s_{12}\right) \alpha_{3}-2 s_{13} \alpha_{1}\right)+\left(s_{39} \alpha_{1}-s_{13} \alpha_{3}\right) 2 \delta_{31} / \delta_{33}}{s_{33}\left(s_{11}+s_{12}\right)-2 s_{13}^{2}}=\lambda,
$$

welche dem Modul der wahren Pyroelectricität proportional ist. Die zur Berechnung des complicirten zweiten Gliedes erforderlichen Zahlenwerthe sind im Vorstehenden zusammengestellt; es erübrigt nur noch die Anwendung der auf p. 1042 angegebenen allgemeinen Werthe der thermischen Dilatationscoefficienten auf die mittlere Beobachtungstemperatur von $22,2^{\circ} \mathrm{C}$, wodurch wir erhalten:

$$
c_{1}=3,36 \cdot 10^{-6}, \quad \alpha_{2}=8,29 \cdot 10^{-6} .
$$

Die Temperatur $22,2^{\circ}$ liegt ausserhalb des von der directen Messung bestrichenen Intervalles; dies dürfte die obigen Zahlen ein wenig unsicher machen, doch jedenfalls nicht so weit, dass dadurch die zu ziehenden Folgerungen irgendwie erschüttert würden.

Die Ausführung der Zahlenrechnung giebt der Gleichung (18) die Form:

$$
645-529=116=\lambda .
$$

Hieraus folgt, dass laut den oben beschriebenen Beobachtungen im Turmalin zwar jedenfalls der grösste Theil der bei einer Erwärmung stattfindenden electrischen Erregung auf eine Wirkung der Deformation zurückgeführt werden muss, dass aber etwa ${ }^{1 / 5}$ als eine directe Wirkung der Temperaturänderung erscheint und somit übrig bleiben würde, auch wenn man durch geeignete Drucke die thermische Deformation aufheben könnte.

Der immerhin geringe Betrag dieses Antheiles scheint mir einigermaassen für die im Eingang erwähnte Vorstellung von dem Mechanismus der electrischen Erregung der acentrischen dielectrischen Krystalle zu sprechen. Jedenfalls stellt sich die wahre und die falsche Pyroelectricität als von so nahe gleicher Grössenordnung dar, dass man darauf hingewiesen wird, eine gemeinsame Ursache für beide anzunehmen; und die oben erörterte schliesst sich wohl am zwanglosesten den allgemeiner adoptirten Anschauungen über die Constitution der Materie an. 
Es mag bemerkt werden, dass das Resultat der von Riecke und mir früher angestellten Beobachtungen mit dem vorstehenden in nächste Uebereinstimmung kommt, wenn man bei der Rechnung statt der Pfaff'schen Dilatationscoefficienten die neu bestimmten benutzt. Man erhält dann in den in jener Arbeit angewandten Einheiten für den Modul der falschen Pyroelectricität den Werth 0,98, während das Mittel der Riecke'schen Beobachtungen für den Modul der gesammten Pyroelectricität 1,23 lieferte. Die Differenz ist wieder ein Fünftel des letzteren Werthes. -

Bei den vorstehenden Entwickelungen ist das Hauptgewicht auf die relativen Werthe der pyro- und piëzoelectrischen Erregungen gelegt; jetzt zum Schluss mag noch beigebracht werden, was sich mit Hülfe der angestellten Beobachtungen über absolute Zahlenwerthe schliessen lässt.

Zunächst giebt der auf p. 1040 angegebene Werth des piëzoelectrischen Moduls $\delta_{33}^{\prime}$ in absolutem Maasse sogleich die Möglichkeit, die Moduln $\gamma$ und $\vartheta$ der ganzen und der wahren pyroelectrischen Erregung in gleicher Weise auszudrücken. Denn $\mu, \nu$ und $\lambda$ sind bez. mit $\delta_{33}^{\prime}, \gamma$ und $\vartheta$ proportional, demgemäss wird sein müssen:

$$
\gamma=\delta_{33}^{\prime} \cdot \frac{\nu}{\mu}, \quad \vartheta=\delta_{33}^{\prime} \frac{\lambda}{\mu} .
$$

Benutzt man die Werthe $\delta_{33}^{\prime}=-0,0567, \nu=645, \mu=30,2$, so erhält man:

$$
\gamma=1,21, \quad 9=0,238 .
$$

Der Werth von $\gamma$ liegt sehr nahe dem Mittelwerth 1,23 aus den unter sich stark abweichenden Moduln, die Riecke bei verschiedenen brasilianischen Turmalinen gefunden hat. ${ }^{1}$ )

Was die allgemeinen Folgerungen aus den vorstehend abgeleiteten Resultaten angeht, so ist zunächst klar, dass, wenn bei einem Krystall das Vorkommen wahrer Pyroelectricität nachgewiesen wird, damit überhaupt die allgemeine Gültigkeit der Curie-Röntgen'schen Hypothese von der vollständigen Zurückführbarkeit der Pyroelectricität auf piëzoelectrische Erregungen umgestossen ist. Dieselbe bleibt zwar unangefochten bei allen Krystallen ohne einzelne krystallographisch

1) E. Riecke u. W. Voigt, l. c. p. 551 u. 552. 
ausgezeichnete Richtungen, wo die Theorie schon allein ausreicht, sie zu beweisen. Bei den übrigen Krystallen ist aber die Berücksichtigung der directen Wirkung einer Temperaturänderung neben derjenigen der Deformationen erforderlich; die Entwickelung der Theorie auf dieser allgemeinsten Grundlage habe ich bereits vor vier Jahren gegeben. ${ }^{1}$ )

Bei allen Krystallen, die nur ein Paar einander entgegengesetzter eigenartiger Richtungen zeigen, ist die Complication, welche die Einführung der wahren Pyroelectricität verursacht, eine sehr geringfügige; sie besteht darin, dass in dem Ausdruck für das dielectrische Moment nach der einen jener Richtungen zu den in den Deformationsgrössen $x_{x}, \ldots x_{y}$ linearen Gliedern noch ein in $\tau$ lineares tritt. $\mathrm{Zu}$ diesen Krystallen gehört Turmalin; für ihn würde demgemäss bei Erregungen, welche die Temperatur ändern, an Stelle von (5) das folgende System zu setzen sein:

$$
\left\{\begin{array}{l}
a=\varepsilon_{15} z_{x}-\varepsilon_{22} x_{y}, \quad b=-\varepsilon_{22}\left(x_{x}-y_{y}\right)+\varepsilon_{15} y_{z} \\
c=\varepsilon_{31}\left(x_{x}+y_{y}\right)+\varepsilon_{33} z_{z}+\vartheta \cdot \tau,
\end{array}\right.
$$

wobei $\vartheta$ den oben angegebenen Zahlenwerth besitzt. Aehnlich würde das System (7) übergehen in

$$
\left\{\begin{array}{c}
-a=\delta_{15} Z_{x}-2 \delta_{22} X_{y}, \quad-b=-\delta_{22}\left(X_{x}-Y_{y}\right)+\delta_{15} Y_{z}, \\
-c=\delta_{31}\left(X_{x}+Y_{y}\right)+\delta_{33} Z_{z}-\gamma \tau
\end{array}\right.
$$

wobei

$$
\gamma=\vartheta+2 \alpha_{1} \varepsilon_{31}+\alpha_{3} \varepsilon_{33}
$$

ist, falls $\alpha_{1}$ und $\alpha_{3}$ wieder die thermischen Dilatationscoefficienten bezeichnen. $\gamma$ hat dabei, wie früher, die Bedeutung des Moduls der ganzen pyroelectrischen Erregung; denn bei einer Erwärmung unter constantem Druck sind alle Componenten $X_{x}, \ldots X_{y}$ gleich Null, und obiges System reducirt sich auf

$$
a=0, \quad b=0, \quad c=\gamma \tau .
$$

\section{Resultate.}

1. Die von Riecke und mir früher durchgeführte Vergleichung zwischen der piëzo- und der pyroelectrischen Erregung eines Turmalines hat ein unrichtiges Endresultat er-

1) W. Voigt, Wied. Ann. 55. p. 701. 1895.

Ann. d. Phys, u. Chom. N. F. 66. 
geben, weil die Pfaff'schen Werthe für die thermisehen Dilatationscoefficienten des Turmalines, auf den von uns benutzten Krystall angewandt, nicht der Wirklichkeit entsprechen.

2. Unter Zugrundelegung eines Zahlensystemes, das vollständig an einem und demselben Krystall bestimmt ist, ergiebt sich, dass von der durch eine Erwärmung unter Atmospärendruck hervorgerufenen electrischen Erregung rund 80 Proc. auf die Wirkung der die Erwärmung begleitenden Deformation, d. h. auf Piëzoelectricität zurückzuführen sind und sonach eine falsche Pyroelectricität bilden. Die uibrigen 20 Proc. stellen dagegen eine directe Wirkung der Erwärmung und somit wahre Pyroelectricität dar. Das gleiche Resultat liefern die früheren Beobachtungen von Riecke und mir bei Benutzung der neuen Werthe der thermischen Dilatationscoefficienten.

3. Hieraus ergiebt sich, dass bei allen Krystallen mit einzelnen krystallographisch ausgezeichneten Richtungen eine vollständige Zurückführung der Pyroelectricität auf piëzoelectrische Wirkungen nicht erwartet werden darf.

4. Dagegen zeigt die Theorie, dass bei allen Krystallen, die einzelner ausgezeichneter Richtungen entbehren, die Erwärmung nur insoweit electrisch wirksam ist, als sie Deformationen veranlasst, dass bei ihnen also scheinbare Pyroelectricität sich stets und vollständig auf Piëzoelectricität zurückführen lässt.

\section{Anhang.}

Ein gepresster Turmalin repräsentirt eine Electricitätsquelle, die bestimmte Ladungsgrössen mit grosser Sicherheit und Bequemlichkeit zu liefern vermag. Er bietet sonach ein vortreffliches Hülfsmittel dar, um Electrometer auf Ladungen zu aichen. So lassen sich z. B. die oben besprochenen Beobachtungen auffassen als Bestimmungen der pyroelectrisch erzeugten Ladungen mit Hülfe eines durch die Piëzoelectricität eines Turmalines geaichten Electrometers.

Zieht man noch ein galvanisches Element von bekannter electromotorischer Kraft heran, so kann man auch die Capacität des Electrometers leicht finden. 
Der Zusammenhang zwischen den Ladungen $E_{1}, E_{2}, E_{3}$ und den Potentialen $P_{1}, P_{2}, P_{3}$ dreier in Wechselwirkung stehender Leiter 1, 2, 3 wird bekanntlich durch die Capacitätscoefficienten $C_{h k}$ der Combination vermittelt gemäss den Formeln

$$
\begin{aligned}
& E_{1}=C_{11} P_{1}+C_{12} P_{2}+C_{13} P_{3}, \\
& E_{2}=C_{21} P_{1}+C_{22} P_{2}+C_{23} P_{3}, \\
& E_{3}=C_{31} P_{1}+C_{32} P_{2}+C_{33} P_{3},
\end{aligned}
$$

wobei $C_{l k}=C_{k h}$ ist.

Sind die Leiter 1 und 2 die Quadrantenpaare eines Electrometers inclusive gleicher mit jedem von ihnen verbundenen Leitersysteme, und steht die Nadel angenähert symmetrisch zu beiden, so kann man $C_{11}=C_{22}$ und $C_{13}=\mathrm{C}_{23}$ setzen. Demgemäss wird

$$
E_{1}-E_{2}=\left(C_{11}-C_{12}\right)\left(P_{1}-P_{2}\right),
$$

und es lässt sich die Differenz $C_{11}-C_{12}$ als die Capacität $C$ eines Quadrantenpaares des Electrometers auffassen.

Der Ausschlag $\sigma$ der Nadel ist bei hinreichender Kleinheit desselben mit $P_{1}-P_{2}$, also auch mit $E_{1}-E_{2}$ proportional; z. B. sei $\sigma=k\left(P_{1}-P_{2}\right)$.

Ein axial mit 1 Kilo comprimirter Turmalin liefert nach p. $1040 \quad 0,0567$ absolute Electricitätseinheiten. Da die beiden Belege des Krystalles bei unseren Beobachtungen mit den beiden Quadrantenpaaren verbunden waren, so war dabei $E_{1}-E_{2}= \pm 0,1134$ Einheiten. Dem entsprach nach p. 1054 der Ausschlag von 30,2 Theilen der Scala. Es war somit

$$
0,1134=30,2 \mathrm{C} / \mathrm{k} \text {. }
$$

Waren dagegen die beiden Quadrantenpaare mit den Polen eines Cadmiumelementes von der electromotorischen Kraft 1,024 Volt verbunden, so zeigte die Nadel nach p. 1044 einen Ausschlag von 45,0 Scalentheilen.

Hier galt somit

$$
45,0=\frac{k \cdot 1,024 \cdot 10^{8}}{3 \cdot 10^{10}}=k \cdot 0,314 \cdot 10^{-2} .
$$

Multiplicirt man diese beiden Formeln, so resultirt

$$
C=\frac{0,1134 \cdot 45}{30,2 \cdot 0,341} \cdot 10^{2}=49,5 \text {. }
$$


Die Capacität jedes der Quadrantenpaare des Electrometers mit den angehängten Belegungen der Krystallpräparate $k_{1}$ und $k_{2}$ und den bezüglichen Drahtleitungen ist somit gleich 49,5 cm. Durch besondere Versuche, deren Aufzählung hier unterbleiben kann, liess sich auch das Verhältniss der Capacitäten der einzelnen Theile bestimmen, aus denen sich jener Werth zusammensetzt. Er fand sich für das Quadrantenpaar und die Leitung bis zur Verzweigungsstelle (vgl. Fig. 1) der Werth $32,6 \mathrm{~cm}$, für einen Beleg des erwärmten Krystalles mit der entsprechenden Leitung $10,6 \mathrm{~cm}$, für den des gepressten Krystalles ebenso 6,3 cm.

Göttingen, Anfang Juni 1898.

(Eingegangen 22. October 1898.) 